\title{
The Application of Augmented Reality on Orienteering Tour Competition
}

\author{
Chih-Hung Wu, Huei-Ming Shih, Li-Shan Ma
}

\begin{abstract}
Traditional orienteering tour competitions are fixed on the route and checkpoints of tourist attractions. Some competitors may attempt to cheat on the prizes by teamwork. For example, the competitor reaches the checkpoint first and knows the next checkpoint, then informs other cooperative competitors to go directly to the next checkpoint. This trick is unfair to other competitors. To resolve this problem of the unfairness, the study designs an mobile app of augmented reality (AR) based on marker-less image recognition technology. The competitors must log in to this app with their account and passwords before starting the competition. The app can augment physical real-world environments of the tourist attraction with the historical introduction and the website address of next tourist attraction selected by random on the screen of the smartphone. The competitors obtain above information only from the app before the specific checkpoint of tourist attraction. Otherwise, they need upload a selfie standing in front of the checkpoint. These two anti-fraud mechanisms and augmented reality technology can resolve the unfair problem of orienteering tour competition and combine the tourism marketing.
\end{abstract}

Keywords-Orienteering Tour, Augmented Reality (AR), Marker-Less Image Recognition

\section{Introduction}

Orienteering is a sport in which the competitors use map and compasses to cross unknown terrain and must visit several checkpoints marked on the map in the shortest possible time and optimal route, and finally return to the end point[1]. Orienteering is based on the concept of map utilization and interpretation, and uses a compass as a positioning and orientation tool, so it is an intelligent use activity [2]. Orienteering is one of the official competitions for the hearing impaired Olympics and is currently a popular sport in many countries.

Chih-Hung Wu / Department of Creative Product and Game Design Chienkuo Technology

Taiwan, R.O.C.

Huei-Ming Shih / Department of Kinesiology Health Leisure Studies Chienkuo Technology

Taiwan, R.O.C.

Li-Shan Ma / Department of Electrical Engineering

Chienkuo Technology

Taiwan, R.O.C.
The checkpoints of orienteering are set up at tourist attractions, and which can be famous monuments or hot spots. The competitors can enjoy the beautiful scenery along the way while visiting the checkpoints. When they arrive at the checkpoint of the tourist attraction, they can know the cultural history of the tourist attraction. After the orienteering competition, the competitors can use their free time to taste the delicious food near the tourist attraction. It is a good idea to combine the orienteering with tourist attractions to reach tourism promotion[3].

In some traditional orienteering tour competitions, the competitors go to the next tourist attraction and visit the checkpoint according to the instructions of the previous checkpoint. However, some competitors could cooperate with other competitors for prizes in the competition. For example, the competitor who first goes to the checkpoint will inform other cooperative competitors to go directly to the next checkpoint. This teamwork will make the competition unfair.

To solve the unfair problem of orienteering tour competitions and effectively combine with tourist attractions to reach tourism promotion, the study uses mobile information technology. Due to the heterogeneity, uncertainty, and perishability of tourism, special information is needed, especially for electronic operation[4]. Since the 1990 s, the relationship between information technology and tourism has become a very important topic and academic field. Many scholars have discussed the impact of information and communication technologies (ICT) on the tourism industry and tourism behavior[5].

\section{SYSTEM ARCHITECTURE}

This study selected Changhua City in Taiwan as the venue for the orienteering tour competition, because Changhua City has rich and unique cultural tourist attractions, well-known snacks and excellent public rental bicycle system "YouBike". YouBike is quite suitable for orienteering activities. The study adopts bicycles as a travel tool of orienteering tour competition. The competitors use the augmented reality application designed by the study as an auxiliary tool. The first competitor will win the prize to visit all eight checkpoints of tourist attractions in Changhua City.

This study designs an mobile app of augmented reality (AR) based on marker-less image recognition technology. The app can be executed on the smartphones. The competitors must log in to this app with their account and passwords before starting the competition. When the competitor arrives at the checkpoint of the tourist attraction, the competitor must take a selfie before the checkpoint and upload the selfie to the web server by the app. After that, the camera of the smartphone aims at the checkpoint. When the AR app identifies the checkpoint successfully, it will overlay the introduction of the tourist attraction and the 
Proc. of The Seventh International Conference on Advances in Computing, Electronics and Communication - ACEC 2018 Copyright ( ) Institute of Research Engineers and Doctors, USA. All rights reserved.

ISBN: 978-1-63248-157-3 doi: 10.15224/978-1-63248-157-3-04

URL button of the next checkpoint onto the real-world of the checkpoint on the smartphone screen. The competitors can know cultural history through the introduction of tourist attraction. In addition, the competitors can click the URL button to link to the web server to obtain the content and location map of the checkpoint of the next tourist attraction. After arriving at the next checkpoint of the tourist attraction, the competitors repeat the above process.

The flowchart and architecture of this system are shown in Fig. 1. The major parts of the system include the design of augmented reality(AR) app and dynamic web page, which are described below.

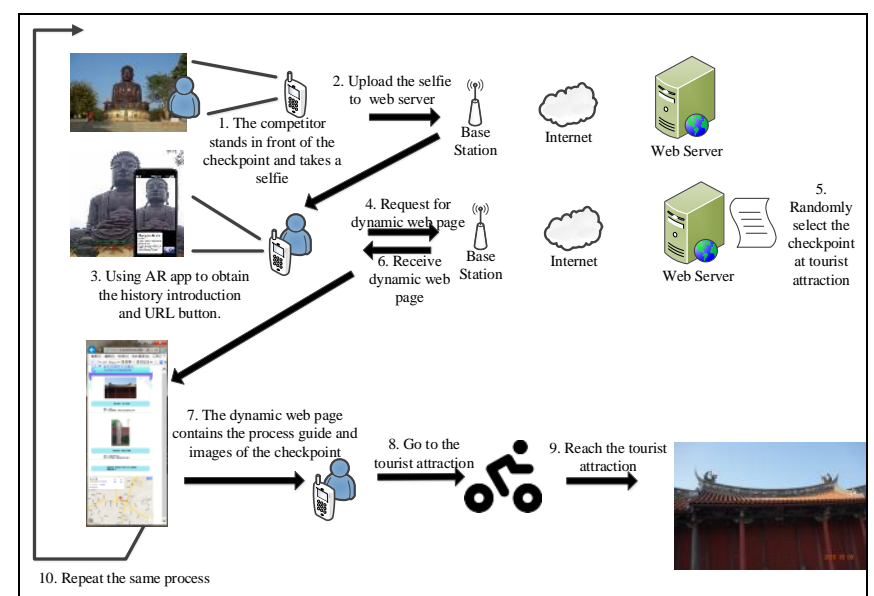

Fig. 1 The flowchart and architecture of this system.

\section{A. Design of Augmented Reality (AR) App}

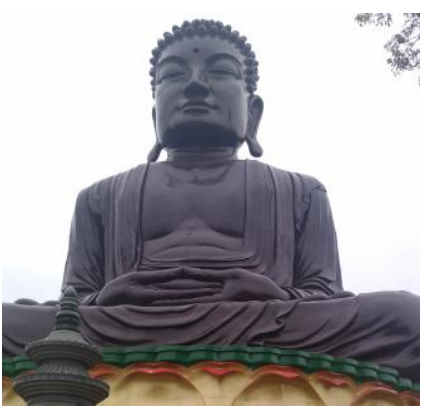

Fig. 2 The appearance image of the checkpoint.

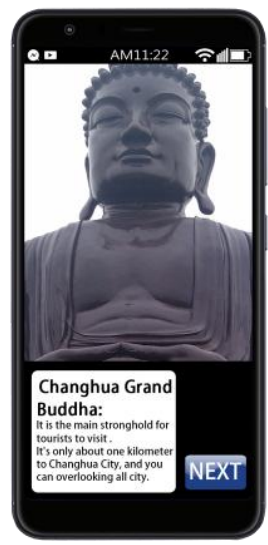

Fig. 3 The appearance image of the AR app.
The study creates augmented reality(AR) app based on the marker-less image recognition technology by the Unity game engine development platform. The Vuforia package designed by Qualcomm Corporation provides the app with required augmented reality components.
The development process is to first take the appearance image of the checkpoint such as Fig. 2. Then upload the image to the database of Qualcomm Corporation's cloud server. The server will use the image to create a maker for image recognition. When the smartphone's camera recognizes this marker in the real world while running the AR app, it will augment the digital objects such as text, graphics and buttons onto the real-world environment on the smart phone screen as shown in Fig. 3.

\section{B. Dynamic Web page}

The dynamic web page in this study is written in PHP programming language. The flow chart of the dynamic web page is shown in Fig. 4. The dynamic web page first displays the image of the next tourist attraction, so the competitors can understand the next destination clear. The study chooses more than two checkpoints in each tourist attraction. The dynamic web page will select one of the checkpoints randomly, so that each competitor may visit different checkpoint in every tourist attraction. The checkpoint selection mechanism can solve the unfair problem of traditional orienteering tour competition.

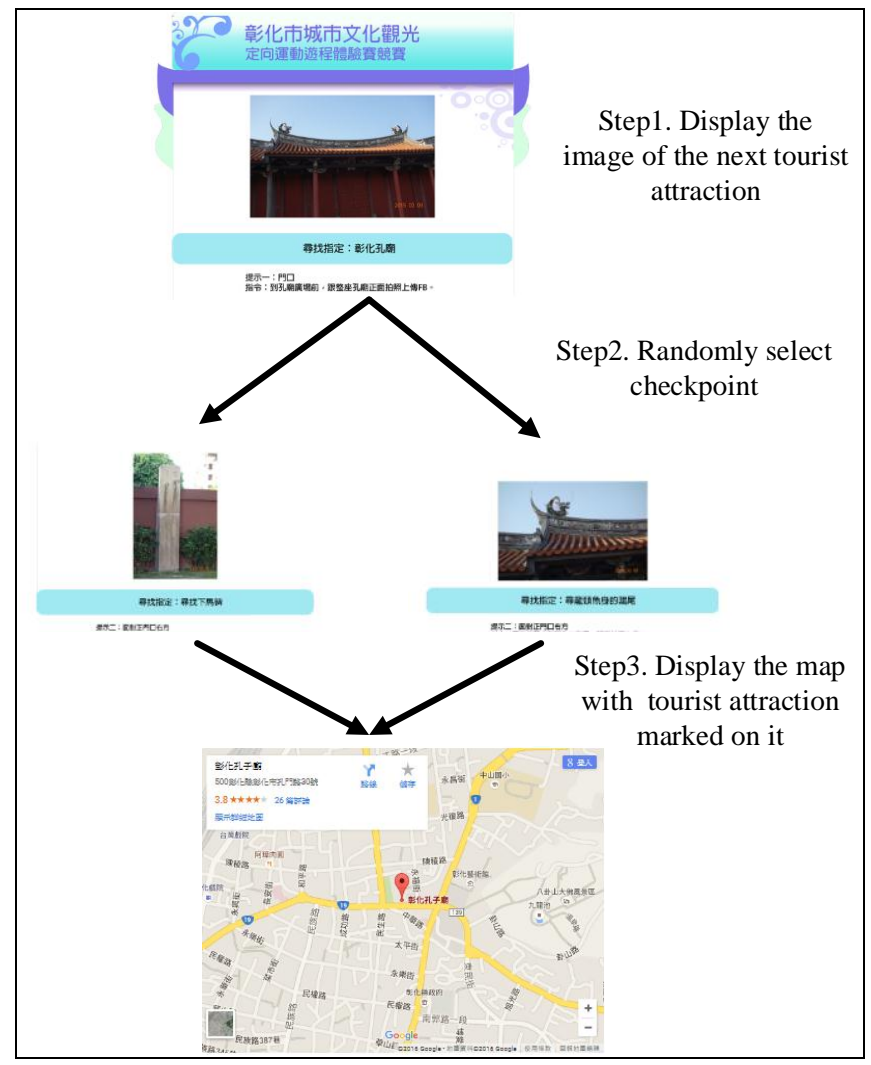

Fig. 4 The flowchart of dynamic web page.

\section{Conclusions}

The augmented reality(AR) app designed by the study can assist the competitors to visit all checkpoints step by step. In the competition, two anti-fraud mechanisms of 
selecting checkpoints randomly by the dynamic web page and uploading the selfie to the web server can solve the unfair problem of teamwork. Through the augmented reality(AR) app, competitors of orienteering tour competition can know the cultural history of every tourist attraction while visiting the checkpoints. It is helpful to combine the orienteering with tourist attractions to reach tourism promotion.

\section{References}

[1] International Orienteering Federation (IOF), “About Orienteering," Retrieved May 13, 2012, from International Orienteering Federation Web site: http://orienteering.org/about-orienteering/

[2] Wen Zhuda and Li Shengtang, "Orienteering and environmental creative teaching applications," Journal of the Republic of China Map Society, Vol. 18, pp. 117-125, 2008.

[3] H.M. Shih, S.L. Chen, and Y.M. Wang, "A Study about Incorporating the Mobile Technology Integration System of an Orienteering Tour into a Sightseeing Tour Planning Curriculum," World Transactions on Engineering and Technology Education, Vol. 14, pp. 271-276, 2016.

[4] Proll , Birgit and Werner Retschitzegger, "Discovering Next Generation Tourism Information System : A Tour on TIScover,' Journal of Travel Research, Vol. 39, pp. 182-191, 2000.

[5] Tierney, P., "Internet-based evaluation of tourism web siteeffectiveness: Methodological issues and survey results," Journal of Travel Research, Vol. 39, pp. 212-219, 2000. 